\title{
Influences on the Work Desire of Cultural Industry Employees-Based on the SEM with the Data of 303 Questionnaires
}

\author{
Juanli Lan ${ }^{1}$, Hongzhen Lei $^{1}$, Junna Sun ${ }^{1}$, Dan Yuan ${ }^{2}$ \\ ${ }^{1}$ International Business School, Shaanxi Normal University, Xi'an, Shaanxi , 710119, China \\ ${ }^{2}$ School of Management, Xi'an University of Architecture \& Technology, Xi'an Shaanxi, 710055, \\ China
}

Keywords: Cultural industry, Stress perception, Social support, Work desire, Structural equation model

\begin{abstract}
Cultural industry is an important part of national economy, but it is still not the mainstay industry of national economy in our country, which is closely connected with the shortage of culture industry talent. Based on the 500 college graduates of Shaanxi province, it explores the relationship among pressure perception,social support and work desire of Cultural industry practitioners, and then checks the moderation of social support. The results show that: stress perception and social support play a significant impact on the work desire of cultural industry personnel employed, and the stress perception is negative related to the work desire, while the social support is positive; social support has moderating effect between stress perception and work desire, low social support plays a negative moderation between them, while the moderation of high social support is not significant.
\end{abstract}

\section{Question Introduction}

"To be a cultural power" is a long-run strategy of cultural development of the Party Central Committee and the State Council of China. The development degree of culture decides the competitive power of a country in global cultural exchanges[1][2]. According to the UNESCO and the GATT, cultural industry is an industry involving the creation, production and distribution of products and services with cultural attributes, which holds an important position in the tertiary industry. It was proposed at the fifth plenary session of the 17th CPC Central Committee to "promote the cultural industry, and make it a pillar industry in national economy". Besides, it was pointed out in the 2013 government work report that the increment in the cultural industry reached $15 \%$, in the amount of RMB210 million based on conservative estimation, which showed that the cultural industry was expected to become the next pillar industry in national economy.

Shaanxi Province is taken as an example. In 2013, the sectors in the cultural industry which had obtained high investment included the cultural leisure and entertainment service (50.7\%), the culture and art service (17.6\%), and the production of culture articles (12.4\%); in respect of employment, the scientific research and technology support service sector provided most job posts (47.0\%), followed by the information transmission, software and information technology service sector (31.6\%), and the culture, sports and entertainment sector (21.3\%). It shows that Shaanxi Province's cultural industry investment focused on the cultural leisure and entertainment service sector. Through analyses, the proportion tendency of job posts provided by each sector in the cultural industry from 1987 to 2013 has been figured out, as shown in Diagram 1. 


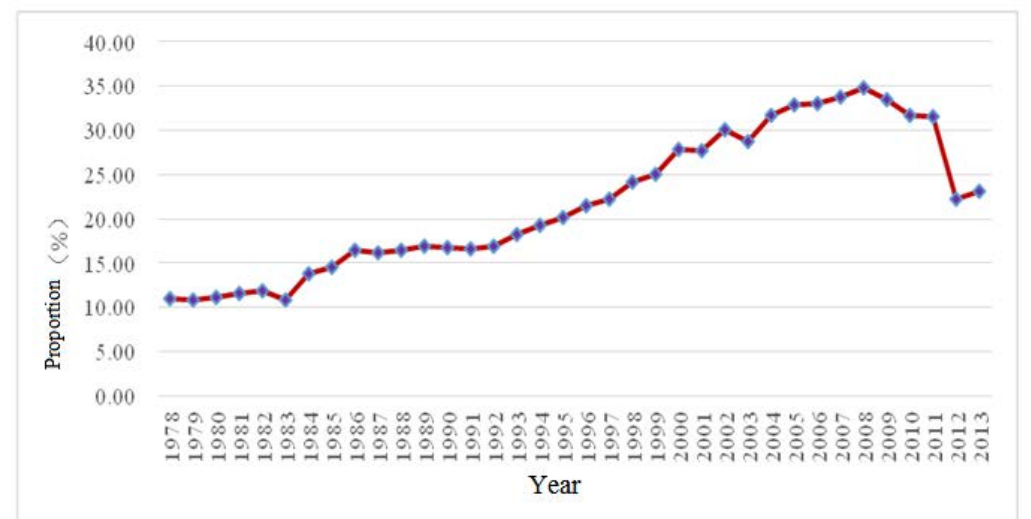

Fig.1. Proportion Tendency of Job Posts of the Cultural industry to that of the Tertiary Industry of Shaanxi Province from 1987 to 2013

According to Diagram 1, it is found that the proportion of job posts provided by the cultural industry takes on a rising tendency. What are the factors affecting the participation desire of practitioners in the cultural industry?

In view of this, it is planned to construct a relevant conceptual model in this article. With the college graduates in Shaanxi Province as an example, a structural equation model (SEM) will be adopted, to discuss their perception of stress, and participation desire in the cultural industry under social support, to draw a constructive conclusion.

\section{Theory and Hypothesis}

\section{Introduction to Conceptual Model}

Through summarizing the existing documents, it is found that the research on the influence factors of the growth of talent in the cultural industry is advancing day after day. For example, LI Yin et al (2014) [3] divided the talents in the cultural industry into four types by value creation: the creative, the operating, the managing, and the content; while Kaufmann.G et al (1997) [4] proposed that "the way that the external factors influencing the growth of the aforementioned four types of talents were basically consistent” long ago. HE Liwen et al (2015) [5] found that, based on the analysis of the input and output efficiency in the cultural industry in 2010 with DEA model, the school enrollments could directly reflect the level of education of residents, and believed that the raising of education level was the key factor that led to the increase of cultural consumption. DENG Yufan et al (2009) [6] have traced out the geographical distribution law of employees engaged in creative industry in Guangzhou through comparative analysis and research of the whole urban space, the urban regional and spatial structure, and the distribution space of different creative sectors; ZHAO Ruifeng et al (2011) [7] have provided a scientific method of forecasting the development of practitioners in the cultural industry in Guangxi by constructing a grey predication model for analyzing the development tendency of practitioners in the cultural industry in Guangxi.

A hypothesis is built as shown in Diagram 2.

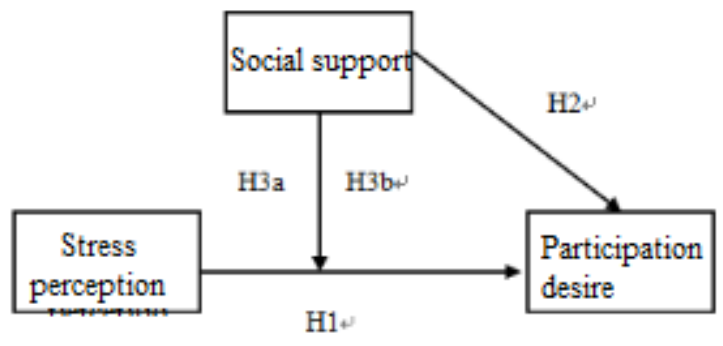

Fig. 2. Research Model 


\section{Stress Perception, Social Support and Participation Desire}

Lazarus et al (1958) [8] believed that stressful event referred to conflict, contradiction, and frustration, and significant changes to people caused thereby. Kaplan et al (1984) [9] proposed that mental stress included the correlation among family, environment and job nature. FAN Fumian and LI Wei (2000) [10] found that most students had an unhealthy psychological status and physiological reaction through search. The research of LIU Jie (2004) [11] showed that $80.1 \%$ of college students obviously felt heavy stress. All these above are responsible for the change of stress perception of the college graduates at the time they decide to enter the cultural industry.

Cullen (1994) [12] believed that social support referred to the emotional and material support given by the society or family or friends to an individual, while Malecki (2002) [13] et al insisted that social support refer to the supporting activities of human body to the people around.

Based on the analyses as above, the following hypotheses are put forward:

$\mathrm{H} 1 \mathrm{Stress}$ perception has a negative impact on the desire of college graduates of participating in the cultural industry.

$\mathrm{H} 2$ Social support has a positive impact on the desire of college graduates of participating in the cultural industry.

\section{Adjustment Function of Social Support}

SHI Jianfeng, YE Junjie, et al believed that as long as an individual could feel social support, such as respect, understanding and satisfaction, the individual would believe in social support [14][15]. QUAN Hongyan et al (2008) [16] found, through survey, that the mental health of college students was a type of throughout social support, which made the individual have a stable emotion and be responsible. GONG Yuxuan (1994) [17] believed, through research, that social support was an important resource for individuals to manage stress. Based on the analyses as above, the following hypotheses are put forward:

H3 Social support can adjust the stress perception and the participation desire, of which the effect is decided by the degree of social support.

The hypothesis as shown above contains two meanings. Thus it can be expressed:

H3a The effect of high social support adjusting college students' stress perception and participation desire is not significant.

H3b Low social support has a negative adjustment effect on college students' stress perception and participation desire.

\section{Research Design}

\section{Sample Selection and Research Tool}

To facilitate sampling, 500 questionnaires had been handed out to the college graduates in Shaanxi Province, and 303 effective questionnaires had been taken back at final. The rate of effective questionnaire reaches $60.6 \%$.For purpose of ensuring the reliability and validity of questionnaire variables, a stress perception assessment scale is adopted for analysis in this article, mainly referring to the CSQ of Pagana, the Dental Environment Strees Questionnaire (DES) of Garbee, etc. The social support is measured from the aspects of "the degree of respect, the degree of understanding, and the degree of satisfaction" based on the subjective support part in the "Social Support Assessment Scale" [18] in the mental health assessment handbook.

\section{Process and Method of Data Analysis}

\section{Reliability Test}

First, according to Cronbach's $\alpha$ analysis of questionnaire with SPSS, it is found that the reliability is high; second, the overall Cronbach's $\alpha$ of stress perception, social support and participation desire all comply with the threshold value $(\alpha>0.7)$ proposed by Nunnally; last, all variables in the scale have been subjected to Cronbach's $\alpha$ check, of which all results are higher than 0.7. This shows that the variables in this research are highly reliable. 


\section{Validity Test}

We have properly modified the questionnaire based on the suggestions given by the subjects of pre-survey, and have verified the construct validity [19] with a structural equation model AMOS7.0 software. According to the results, we draw a conclusion that GFI, CFI, NFI, IFI, RFI, etc. all approximately reach 0.9 , which shows that the construct validity of stress perception is good.

(3) Related Analysis

We have tested whether the variables are closely related to each other via correlation coefficient. If the test result reaches 0.05 ( $\mathrm{p}<0.05$ ), the correlation coefficient will be marked with “*”; if reaches $0.01(\mathrm{p}<0.01)$, then marked with “**“. According to Table 1, the prerequisites for testing the regulated variables have been met with.

Table 1. Mean Value and Standard Deviation of Participation Desire, Stress Perception and Social Support and Related Analysis thereof

Participation DesireStress PerceptionSocial Support

\begin{tabular}{cccc}
\hline Participation Desire & & \\
\hline Stress Perception & $0.357^{* *}$ & 1 & \\
\hline Social Support & $0.256^{*}$ & $0.518^{* *}$ & 1 \\
\hline Mean Value & 5.0112 & 103.7830 & 18.9083 \\
\hline Standard Deviation & 2.71947 & 16.19382 & 3.28792 \\
\hline
\end{tabular}

\section{Model Empirical Research and Analysis}

\section{Confirmatory Factor Analysis}

According to the empirical analysis results, the professional skill contributes most, of which the factor loading is 0.73 , followed by workload, professional experience, employment status, interpersonal relationship and educational background; while among the three indexes of social support, the degree of understanding contributes most, of which the factor loading is 0.57 , followed by the degree of satisfaction (0.48) and the degree of respect (0.39). After correcting the model in accordance with the standard of fit index, both CFI and IFI of the fitness degree are higher than 0.90. This indicates that the fitness of the model is good as a whole, as shown in Tables 2 and 3.

Table 2. Confirmatory Factor Analysis Index of Stress Perception

\begin{tabular}{llllllllllll}
\hline & $x^{2}$ & df & $x^{2} / \mathrm{df}$ & GFI & CFI & IFI & RFI & NFI & AIC & BCC & ECVI \\
\hline $\mathrm{M}_{0}$ & 58.967 & 9 & 6.552 & 0.878 & 0.841 & 0.851 & 0.708 & 0.819 & 90.698 & 92.507 & 0.456 \\
& 8.329 & 7 & 1.190 & 0.988 & 0.989 & 0.997 & 0.960 & 0.983 & 35.898 & 36.575 & 0.179 \\
$\mathrm{M}_{1}$ & & & & & & & & & & & \\
\hline
\end{tabular}

Note: $\mathrm{M}_{0}$ before model modification; $\mathrm{M}_{1}$ after model modification

Table 3. Regulatory Effect Model of Social Support

\begin{tabular}{llllllllllll}
\hline & $\mathrm{x}^{2}$ & $\mathrm{df}$ & $\mathrm{x}^{2} / \mathrm{df}$ & $\mathrm{GFI}$ & $\mathrm{CFI}$ & $\mathrm{IFI}$ & $\mathrm{RFI}$ & $\mathrm{NFI}$ & AIC & BCCQ & ECVI \\
\hline $\mathrm{M}_{0}$ & 259.797 & 74 & 3.511 & 0.816 & 0.715 & 0.698 & 0.563 & 0.668 & 332.167 & 326.965 & 1.662 \\
& & & & & & & & & & & \\
$\mathrm{M}_{1}$ & 92.236 & 64 & 1.442 & 0.943 & 0.967 & 0.961 & 0.835 & 0.875 & 172.674 & 181.171 & 0.869 \\
\hline
\end{tabular}

Note: $\mathrm{M}_{0}$ before model modification; $\mathrm{M}_{1}$ after model modification

\section{Result Analysis}

We have constructed the model immediately after making confirmatory factor analysis over the data with AMOS7.0 software, as shown in Diagram 3. The diagram shows that the more sensitive the stress perception is, the more reluctant the college graduates are to enter the cultural industry, namely the lower the participation desire; the impact of social support on participation desire is not significant; the interaction item edge between stress perception and social support significantly have a negative impact on participation desire.

The adjustment effect of social support on the correlation between stress perception and participation desire is not significant, as shown in Diagram 4. The diagram shows that high social support can not significantly adjust college graduates' stress perception and participation desire. 


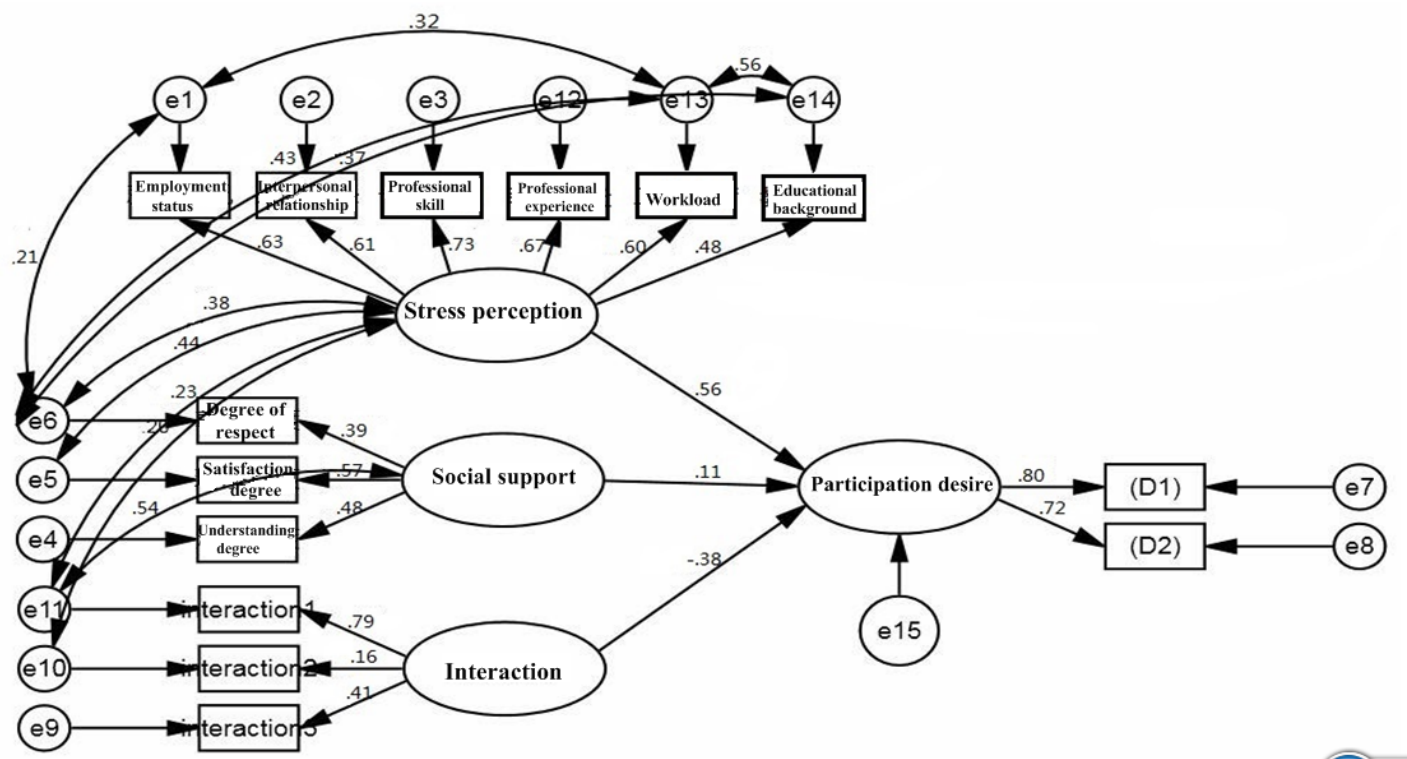

Fig. 3. Modified Model

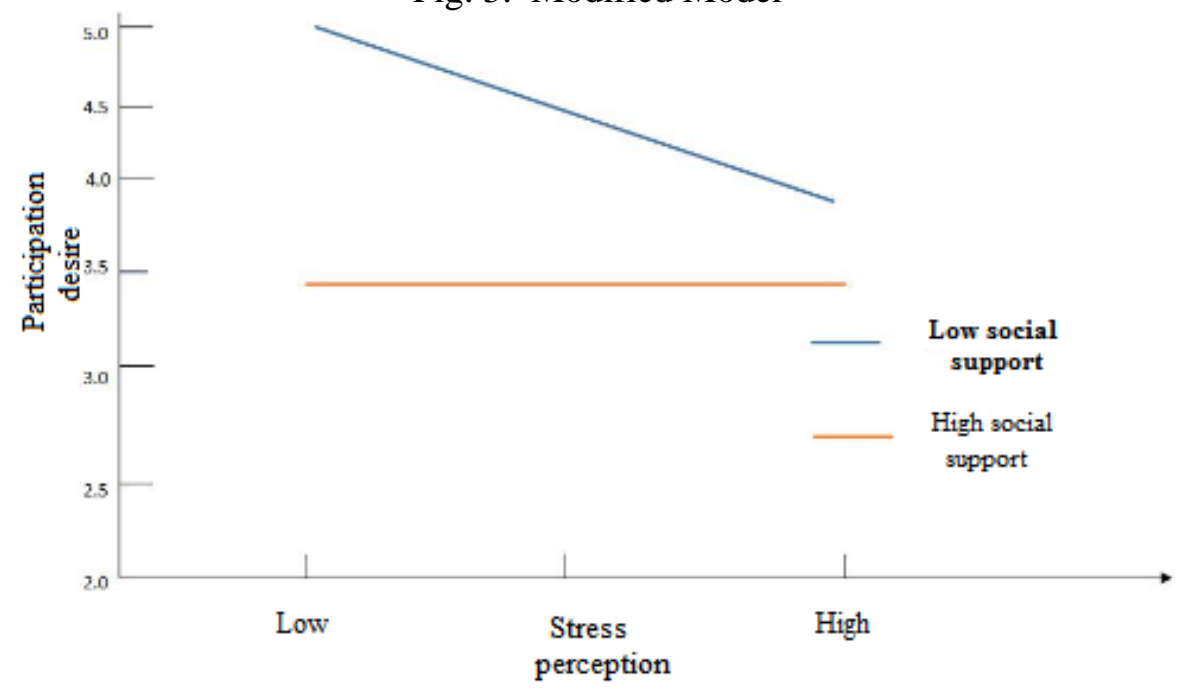

Fig. 4. Regulation Function Sketch of Social Support

\section{Conclusion}

In this research, the five hypotheses all are proved to be tenable through the analysis of the correlation among the three variables in the conceptual model constructed by integrating the stress perception, social support and participation desire of the practitioners in the cultural industry into a research framework, and the correlation among the stress perception, social support and participation desire of the practitioners in the cultural industry proposed in this article is also proved to be tenable on the whole. On this basis, the following suggestions have been put forward on how to promote the cultural industry and arouse the enthusiasm of the practitioners in the cultural industry, as below: 


\section{Reduce stress}

Help the practitioners in the cultural industry reduce stress at the source, to raise their participation desire, and attract more persons to enter the cultural industry. First, the practitioners themselves should enhance their knowledge; second, the government and enterprises should make efforts to improve the employment status of the practitioners in the cultural industry, so that more culture practitioners can make contributions according to their ability.

\section{Provide more social support to a proper extent}

The prosperity and development of the cultural industry requires more efforts to cultivate professionals by reasonably, purposefully and designedly developing and cultivating human resources for the cultural industry. As analyzed in the article, social support should remain at a proper level. McClelland pointed out in the achievement need theory that the respect to and the recognition of their achievements [20] are the best reward for those who lay emphasis on achievement need. Therefore, social support should be controlled within a reasonable range.

\section{Expand employment channel}

The government should give support by promoting the coupling development between the cultural industry and its related industries, and expanding employment channel while pointing a way to finance to cultural enterprises, to realize the diversified development of the cultural industry.

The strategy of "One Belt and One Road” of China aims to substitute the division mode of labor in traditional industries with a modern, comprehensive and sustainable mode established via cooperation with countries along the line. To this end, with the support of the policy of "One Belt and One Road", we should bring our comparative superiority in the cultural industry into full play, to blaze a trail for realizing the goal of "accelerating economic transition and upgrading of our country".

\section{Acknowledgement}

This article is a national social science fund project, of which the title is: Research on the Diffusion Mechanism, Convergence, and Risk Control of Dissemination of Negative Information among Network Groups, and the project number is: 14BSH052; it is also a project under the National Natural Science Foundation of China, of which the title is: Research on Multi-stage Cluster Sampling Method for Social Commercial Embedding Relationships, and the project number is: 71401092.

\section{References}

[1] HU Shada, WANG Hui, WANG Zhiyuan, LI Xiaoying: Research on Organic Integration of Culture and Technological Innovation under the Strategic Background of Innovation Driving Development, Science and Technology Management Research, 2014, edition 22.

[2] ZHU Yuezhao, DAI Shuchun, CHEN Hongxi: Research on Construction of Technology Innovation System of Scientific and Technological Enterprises under Innovative Perspective, Science and Technology Management Research, 2015, edition 2.

[3] LI Yin, LIU Fang: Research on Influence Factors of Growth of Talents in Cultural industry, Academic Research of Shijiazhuang Institute of Technology, 2014, edition 4.

[4] KAUFMANN. G, VOSBURG.S.K. Paradoxical mood effects on creative problem solving. Cognition and Emotion, 1997(11):151-177.

[5] HE Liwen, YUAN Xiaoling, DENG Minhui: Research on Input and Output Efficiency of Cultural industry in the Top Ten Metropolitan Areas in China, Statistics\&Decision, 2015, edition 1.

[6] DENG Yufan, CHEN Zhongnuan, XIAO Yanfei: Research on Geographical Distribution of Practitioners of Creative Industry in Guangzhou City, Human Geography, 2009, edition 2. 
[7] ZHAO Ruifeng, ZENG Ningfeng, LIU Huaming: Analysis of Development Tendency of Practitioners of Cultural industry based on GM $(1,1)$ Model, China Market, 2012, edition 15.

[8] LAZARUS R S, DELONGIS A, FOLKMAN S, GRUEN R.Stress and adaptational outcomes: The problem of confounded mearsures. American Psychologist,1985,(40):770-779.

[9] KAPLAN, PAUL S., JEAN STEIN. Psychology of adjustment. Thomson Brooks/Cole, 1984.

[10]FAN Fumian, LI Wei: Mental Stress of College Students and Stress Management Method-Survey in Tsinghua University, China Youth Study, 2000, edition 6.

[11]LIU Jie: Common Psychological Problems of College Students and Intervention Strategy, Social Sciences Journal of Universities in Shanxi, 2004, edition 3.

[12]CULLEN F T. Social support as an organizing concept for criminology : Presidential address to the academy of criminal justice, Justice Quarterly,1994,11:527-559.

[13]MALECKI C K, DEMARAY M K. Measuring perceived social support, development of the child and adolescents social support scales. Psychology in the School.2002,39:1-18.

[14]SHI Jianfeng, MA Jianhong: Discussions on Problems in Social Support Research, Chinese Journal of Ergonomics, 2003, edition 1.

[15]YE Junjie: Research on Factors Influencing College Students' Comprehending Social Support, Journal of Psychological Science, Psychological Science, 2005, edition 6.

[16]QUAN Hongyan, LONG Fen, HONG Dongmei, et al: Correlation between College Students' Mental Health and Personality and Their Comprehending of Social Support, China Journal of Health Psychology, 2008, edition 2.

[17]GONG Yuxuan: Overview of Research on Correlation between Social Support and Health, Psychology Dynamics, 1994, edition 2.

[18]WANG Xiangdong, WANG Xilin, MA Hong: Mental Health Assessment Scale Manual, Chinese Mental Health Journal, 1999, edition13.

[19]WU Yan, WEN Zhonglin, HOU Jietai, Marsh Herbert: Standardized Estimation of Interaction Effect Model with No Mean Structure for Adjustment of Latent Variables, Acta Psychologica Sinica, 2011, edition 10.

[20]HAO Dongmei, LI Zhongying: Research on Virtual Human Resource Management Personnel Incentive based on Psychological Contract-Knowledge-based Employee, Science and Technology Management Research, 2013, edition 21. 